NSF-ITP-93-78

UMHEP-387

gr-qc/9307014

July 27, 2021

\title{
Testing Cosmic Censorship with Black Hole Collisions
}

\author{
Dieter R. Brill and Gary T. Horowitz \\ Institute for Theoretical Physics \\ University of California \\ Santa Barbara, CA. 93106 \\ David Kastor and Jennie Traschen \\ Department of Physics and Astronomy \\ University of Massachusetts \\ Amherst, Massachusetts 01003
}

\begin{abstract}
There exists an upper limit on the mass of black holes when the cosmological constant $\Lambda$ is positive. We study the collision of two black holes whose total mass exceeds this limit. Our investigation is based on a recently discovered exact solution describing the collision of $Q=M$ black holes with $\Lambda>0$. The global structure of this solution is analyzed. We find that if the total mass is less than the extremal limit, then the black holes coalesce. If it is greater, then a naked singularity forms to the future of a Cauchy horizon. However, the horizon is not smooth. Generically, there is a mild curvature singularity, which still allows geodesics to be extended. The implications of these results for cosmic censorship are discussed.
\end{abstract}




\section{Introduction}

It is almost 25 years since Penrose first proposed his cosmic censorship conjecture [1]. Roughly speaking, this conjecture states that naked singularities do not form from realistic physical processes. This conjecture is widely believed to be true and has become the cornerstone of our understanding of gravitational collapse and black holes. But despite extensive work over the years, we are still far from having a proof. One appears to need global existence results for strong-field, nonsymmetric solutions, which are extremely difficult to obtain.

When a proof seems difficult, it may be easier to obtain a convincing counterexample and show that the conjecture is false. Many attempts have been made. One of the earliest is the following. Static charged black holes in the Einstein-Maxwell theory are characterized by their mass $M$ and charge $Q$, and exist only for $Q \leq M$. For $Q>M$ the spacetime describes a naked singularity. One can ask whether it is possible to start with a black hole with $Q=M$, drop in a test particle with charge greater than its mass, $q>m$, and turn the black hole into a naked singularity. Wald showed that this cannot happen [2]. In order that a $q>m$ test particle may reach the horizon, it must be thrown in with sufficient kinetic energy that the mass of the black hole increases more than its charge.

In the presence of a positive cosmological constant $\Lambda$, there is a variation of this test. Charged black holes in a de Sitter background have three horizons, an inner and outer black hole horizon, and a de Sitter horizon. There are thus two extremal limits. One corresponds to coinciding inner and outer black hole horizons, and is analogous to the extremal Reissner-Nordström solution. The other corresponds to coinciding outer black hole and de Sitter horizons. In both cases, exceeding these extremal cases results in naked singularities. For the first type of extremal limit, it was shown in [3] that it is again impossible to destroy the black hole by sending in charged test particles. However, we will show in Sec. 2 that for the second type of extremal black hole, one can drop in a charged test particle and exceed the limiting value. One thus seems to have a potential violation of cosmic censorship 
when $\Lambda>0$.

To analyze this situation further, one needs to go beyond the test particle approximation and include the backreaction of the test particle on the geometry. Ideally one would like to have an exact solution describing two black holes colliding in de Sitter space. Remarkably, a solution of exactly this type has recently been found [3]. The solution describes an arbitrary number of $Q=M$ black holes with $\Lambda>0$. The solution is dynamical, and the black holes collide in the future. (Alternatively, one can consider the time reverse, which describes white holes splitting and expanding.) For small $M$, the single $Q=M$ black hole is not extremal and has three horizons. However, when one increases $M$, there is an extremal value where the outer black hole and de Sitter horizons coincide. One can thus consider two black holes each with mass less than this extremal value, but whose sum is greater, and let them collide. The purpose of this paper is to examine this process and determine whether cosmic censorship is indeed violated.

In order to settle this question, several preliminary steps are necessary. Some of these have interesting applications independent of cosmic censorship. First, one needs to investigate the global structure of the multi-black-hole solutions. This requires extending beyond the coordinate patch in which the solutions were first presented. Here we find a surprise. The horizons across which one must extend are in general not smooth, but have only finite differentiability. As a result, the extensions are not unique. This has implications for the instability of the inner black hole horizon $[4,5]$, and yields a possible analogy to the lack of smoothness of null infinity in asymptotically flat spacetimes [6]. Physically, for certain horizons, the lack of smoothness can be interpreted as the result of electromagnetic and gravitational radiation in the spacetime which is not smooth at the horizon. We also study null geodesics and determine the global event horizon. We find that when the total mass is less than the extremal limit, the black holes coalesce.

Since $\Lambda>0$, these solutions are not asymptotically flat, and the usual definitions of black holes and naked singularities are no longer valid. However, for 
spacetimes that asymptotically approach de Sitter, there is an analog of future null infinity, and one can define the event horizon to be the boundary of the past of this null infinity. Since the singularities of a charged black hole are timelike, they are always locally naked. However, if they are enclosed inside an event horizon, there is no violation of weak cosmic censorship. We will be interested in whether the singularity is visible to all observers who start from a given asymptotic de Sitter region in the past. If so, there would be no way for these observers to avoid seeing the singularity, and even weak cosmic censorship would be violated.

Several properties of black holes in de Sitter space have recently been studied by Shiromizu et. al. [7]. They discuss black hole collisions, but without the benefit of an exact solution. By assuming cosmic censorship, they are lead to the conclusion that large black holes will not collide when $\Lambda>0$. We will see that the exact solution behaves quite differently.

By studying this solution, we find that the question of whether cosmic censorship is violated by charged black hole collisions is rather subtle. Three different issues must be dealt with. First, since the total mass exceeds the extremal limit, there is always a naked singularity "on the other side" of de Sitter space, even before the black holes collide. Thus, one is essentially starting with singular initial conditions and should not be surprised if cosmic censorship is violated. We will show that this problem can be alleviated by introducing a charged shell of dust, which will remove the unwanted singularity. Second, there is always a Cauchy horizon, which we will show is generically singular. However, the singularity is rather mild, and geodesics can pass through it. Furthermore, there is a large subset of the initial data for which the curvature singularity is removed. In these cases, all observers cross the Cauchy horizon and see a naked singularity. Cosmic censorship is violated. However, the known exact solutions do not describe the most general black hole collision with a positive cosmological constant. It is certainly possible that in the generic collision the singularity at the Cauchy horizon will be much worse, and there will be no way to continue the spacetime. Finally, the naked singularity appears only for eternal black holes. We will see that one can form these 
black holes from regular initial conditions (e.g. using charged dust). But in this case, the matter forming the black holes and the matter introduced to remove the unwanted singularity collide before a naked singularity is reached. We do not have the exact solution beyond this point, and so do not know if naked singularities will form in the future.

Analysis of the global structure of the multi-black-hole solutions is complicated by the fact that, in general, they are neither static, nor spherically symmetric. In light of this, we begin in Sec. 2 by discussing the special case of a single black hole. This is described by the Reissner-Nordström-de Sitter solution. We will show that, in the test particle approximation, a $Q=M$ black hole can have its mass increased past the extremal limit of coinciding black hole and de Sitter horizons. In Sec. 3 we start our discussion of the multi-black-hole solutions by examining the basic features of their geometry. By locating the trapped surfaces, we see here the first evidence that some of them describe coalescing black holes. In Sec. 4 we investigate extensions of the solutions and discuss the lack of smoothness at the horizons. Sec. 5 contains a discussion of the event horizon and establishes that (when the total mass is less than the extremal limit) its topology indeed changes, showing that black holes do combine. In Sec. 6 we consider the introduction of charged dust. Finally, in Sec. 7, the possible counterexample to cosmic censorship is studied. Sec. 8 contains some concluding remarks.

\section{The $Q=M$ Reissner-Nordström-de Sitter Solution}

The Reissner-Nordström-de Sitter (RNdS) solution is static and spherically symmetric, and its global structure can be analyzed by general methods. This has been done by Brill and Hayward [8]. Here we will briefly recount their results for the case $Q=M$. We also discuss briefly the motion of test particles in $Q=M$ RNdS backgrounds. We show that one can add $q=m$ test particles to an extremal black hole, causing it to exceed the extremal limit. 


\subsection{Static Coordinates}

The static form of the $Q=M$ RNdS metric and electromagnetic potential is

$$
\begin{gathered}
d s^{2}=-V(\tilde{R}) d T^{2}+\frac{d \tilde{R}^{2}}{V(\tilde{R})}+\tilde{R}^{2} d \Omega^{2}, \\
V(\tilde{R})=\left(1-\frac{M}{\tilde{R}}\right)^{2}-\frac{\Lambda}{3} \tilde{R}^{2}, \quad A_{T}=-\frac{M}{\tilde{R}} .
\end{gathered}
$$

We assume $\Lambda>0$, and will interpret $M$ as the mass of the black hole. There is a curvature singularity at $\tilde{R}=0$. Horizons occur where $V(\tilde{R})=0$ and $\tilde{R}>0$. The number of horizons depends on the mass and cosmological constant. For $M^{2} \Lambda<\frac{3}{16}$, there are three horizons: inner and outer black hole horizons and a de Sitter horizon. If the mass of the black hole is increased, the outer black hole and de Sitter horizons move closer together. They coincide at the extremal mass given by

$$
M_{\text {ext }}^{2} \equiv \frac{3}{16 \Lambda} .
$$

For $M>M_{\text {ext }}$ there is only a single horizon. We will call this the "overmassive" case.

The Penrose diagrams for these three cases are shown in Figs. (1 - 3). Fig. (1) shows the case $M<M_{\text {ext }}$ with its three horizons. Notice that $\Im^{ \pm}$are spacelike when $\Lambda>0$. The overmassive case is shown in Fig. (3), and clearly has naked singularities. However, these singularities exist for all time, and hence this is not a violation of cosmic censorship. The extremal limit, Fig. (2), is rather unusual. This solution has singularities, which are visible from $\Im^{+}$, and also a nonsingular spacelike surface to their past. (By making an appropriate identification this surface can even be compact.) However, there are complete timelike geodesics along which the singularity is never visible. (These reach the point $p$ in the figure.) Thus, one can view the point $p$ as a future timelike infinity and define a horizon to be the boundary of its past. This illustrates the difficulty of defining a naked singularity 
when the cosmological constant is nonzero. As stated earlier, we will call a singularity naked if it is visible to all observers originating from a given asymptotic de Sitter region in the past. So the extremal limit is not naked in our sense.

\subsection{Cosmological Coordinates}

The $Q=M$ RNdS solution can also be expressed in isotropic or "cosmological" coordinates:

$$
d s^{2}=-\frac{d \tau^{2}}{U^{2}}+U^{2}\left(d r^{2}+r^{2} d \Omega^{2}\right), \quad U=H \tau+\frac{M}{r}, \quad A_{\tau}=\frac{1}{U}
$$

where $H= \pm \sqrt{\Lambda / 3}$. The transformation from the static coordinates to cosmological coordinates is given by

$$
\tilde{R}=H \tau r+M, \quad T=\frac{1}{H} \log H \tau-h(\tilde{R}), \quad \frac{d h}{d \tilde{R}}=-\frac{H \tilde{R}^{2}}{(\tilde{R}-M) V(\tilde{R})}
$$

Since (2.3) is the form of the solution that is easily generalized to several masses, it is useful to understand how these cosmological coordinates cover the spacetime. In Fig. (1), a single patch of $(r, \tau)$ coordinates with $H<0$ covers the region enclosed by the bold lines. Some constant- $\tau$ surfaces have been drawn in this region. Below the dashed line $\tau$ is negative. The left-hand end of the constant $\tau$ surfaces is $r=\infty$. The right-hand end is $r=0$ (but we will soon see that this is an "infinite throat," not a regular origin of polar coordinates). Above the dashed line $\tau$ is positive. In this region, the right-hand end of the constant- $\tau$ surfaces is still $r=0$, but the chart ends on the left at a finite $r$, where $U$ vanishes and the geometry is singular. Note that this coordinate patch covers a past de Sitter horizon and the inner and outer black hole horizons. The future de Sitter horizon corresponds to $r=\infty, \tau=0$, and the region beyond this horizon, where $\Im^{+}$lies, is not covered by these coordinates, but it is covered by (2.3) with $H>0$.

The extremal limit and overmassive cases, Figs. (2) and (3), are similar. Again we have drawn some constant- $\tau$ surfaces and again $\tau=0$ is shown as a dashed 
line. The geometry of the spatial slices for $\tau<0$ is regular, but for $\tau>0, r$ again extends only to a finite maximum value at the singularity.

\subsection{Paths of Test Particles}

Consider a $q=m$ test particle with conserved energy $E=m$ moving in a $Q=M \mathrm{RNdS}$ spacetime. It was shown in [3] that the radial motion, in static coordinates, is given by

$$
\frac{d \tilde{R}}{d \lambda}= \pm H \tilde{R}, \quad \frac{d T}{d \lambda}= \pm \frac{1}{V(\tilde{R})}\left(1-\frac{M}{\tilde{R}}\right)
$$

where $\lambda$ is the proper time along the path. There are two possibilities for the motion depending on whether the signs in (2.5) are chosen to be the same or opposite. The other choices of sign in (2.5) reverse the flow of time along these paths. It is simple to check, using (2.4), that choosing both plus signs in (2.5) gives a path of constant $r$ in cosmological coordinates. Similarly, we can define a new set of cosmological coordinates $\left(r^{\prime}, \tau^{\prime}\right)$ by reversing the sign of the static time coordinate $T$ in (2.4). The paths (2.5), with opposite choices of signs are then paths of constant $r^{\prime}$. These paths of constant $r^{\prime}$ will be important to us in Sec. 6 when we discuss the collapse of charged shells.

Now consider a test particle on a path of constant $r$, such as the one shown in Fig. (1). At early times the test particle is outside both the de Sitter and black hole horizons. As time progresses, it enters first the de Sitter and then the black hole horizon. Nothing in the above discussion relied on the black hole being undermassive. If the black hole is an extremal one, the description is roughly the same, with the test particle passing through the degenerate horizon. It follows immediately that there is no barrier to such a test particle entering the horizon. One can exceed the extremal mass limit by dropping in test particles. Furthermore, since the charges on the black hole and test particle provide a repulsive force, we expect that this phenomenon will be generic for black holes and test particles with charge less than their mass. 


\section{General Properties of the Multi-Black-Hole Solutions}

\subsection{Geometry of SPatial surfaces}

The solutions of reference [3] depend on a number of parameters that correspond to several different masses at arbitrary positions (but not arbitrary velocities). The metric and gauge field for mass parameters $M_{i}$ and positions $\vec{r}_{i}$ are given by

$$
\begin{array}{rlrl}
d s^{2} & =-\frac{d \tau^{2}}{U^{2}}+U^{2} d \vec{r} \cdot d \vec{r}, & A_{\tau}=\frac{1}{U} \\
U & =H \tau+\sum_{i} \frac{M_{i}}{\left|\vec{r}-\vec{r}_{i}\right|}, & H & H= \pm \sqrt{\Lambda / 3} .
\end{array}
$$

Unless otherwise stated, we will assume $H<0$. It is only for this case that the spacetime describes black hole collisions. In terms of $H$, the extremal limit (2.2) is

$$
M_{e x t}=\frac{1}{4|H|}
$$

The surfaces of constant $\tau$ are spacelike everywhere. Near each $\vec{r}_{i}$ the geometry resembles the infinite throat familiar from the asymptotically flat extremal Reissner-Nordström solution. This can be seen by expressing the spatial metric in spherical coordinates centered at $\vec{r}=\vec{r}_{i}$. Near the origin of these coordinates, this metric becomes

$$
d l^{2} \approx \frac{M_{i}^{2}}{r^{2}} d r^{2}+M_{i}^{2} d \Omega^{2}
$$

which is the metric for a cylinder of infinite spatial extent having cross sectional area $4 \pi M_{i}^{2}$.

The curvature of (3.1) can be singular at zeros of the metric function $U$. This can be seen from the square of the Maxwell field strength,

$$
F^{2} \equiv F_{\mu \nu} F^{\mu \nu}=-\frac{(\vec{\nabla} U)^{2}}{U^{4}}
$$

where $\vec{\nabla}$ denotes the usual gradient with respect to $\vec{r}$. If $U=0$ and $\vec{\nabla} U$ does not 
vanish like $U^{2}$ or faster, then $F^{2}$ diverges and the curvature is singular. The metric is regular everywhere else, but it is incomplete as discussed in Sec. 4.

Assembling these elements we get a picture of how the spatial geometry develops in cosmological time. We will start with one black hole first. Consider the spatial surfaces for a single black hole with mass $M<M_{\text {ext }}$, which are sketched on

Fig. (1). The metric function is $U=H \tau+\frac{M}{r}$. For $\tau<0, U$ is positive everywhere and the spatial surfaces are nonsingular. They are asymptotically flat and have the cylindrical form of an infinite throat near the origin. For $\tau=0$, the spatial metric is regular and has the cylindrical form everywhere. As $\tau$ is increased slightly, a singularity appears near $r=\infty$. As $\tau$ increases further, the singularity cuts off more and more of the cylinder. In Fig. (1) this is shown by the way the singularity intersects the spatial surfaces.

The generalization to more than one mass is then straightforward. For $\tau<0$ the spatial surfaces are nonsingular and asymptotically flat at large radius. Near each "point" $\vec{r}_{i}$, the spatial metric has the form (3.3) of a throat. These surfaces are depicted in Fig. (4a). For $\tau=0$, the surface is nonsingular, but spatial infinity is now also asymptotically cylindrical. This surface is depicted in Fig. (4b). As $\tau$ is increased slightly above zero, a singularity moves in from spatial infinity as shown in Fig. (4c). As $\tau$ continues to increase, the singularity splits and eventually surrounds each of the throats individually. The spatial surface is then composed of a number of isolated throats, as depicted in Fig. (4d). This description is independent of the size of the masses, and hence applies to both the overmassive and undermassive cases.

\subsection{TRAPped Surfaces}

The causal structure of the multi-black-hole solutions is rather involved. To gain some understanding, we begin by considering trapped surfaces. The expansions $\theta_{\text {out }}\left(\theta_{\text {in }}\right)$ of outgoing (ingoing) null rays normal to a 2 -surface $\Sigma$ in a spatial 
hypersurface are given by

$$
\theta=D_{i} n^{i}-K_{i j} n^{i} n^{j}+K
$$

where $h_{i j}, K_{i j}$ are the metric and extrinsic curvature on the spatial hypersurface, $D_{i}$ is the covariant derivative compatible with $h_{i j}, K=h_{i j} K^{i j}$, and $n^{i}$ is the outward (inward) directed normal vector to $\Sigma$. A surface $\Sigma$ is called outer trapped if $\theta_{\text {out }}<0$, and inner trapped if $\theta_{\text {in }}>0$. Surfaces for which $\theta_{\text {out }}\left(\theta_{\text {in }}\right)$ vanish are called outer (inner) apparent horizons. For the surfaces of constant $\tau$ in the metric (3.1), the extrinsic curvature is simply given by $K_{i j}=H h_{i j}$.

In the case of a single black hole of mass $M$, one can calculate the expansions exactly for spheres centered on the origin. The result depends only on the quantity

$$
R \equiv H r \tau,
$$

which is simply related to the static $\tilde{R}$ coordinate by $R=\tilde{R}-M(2.4)$. The expansions are

$$
\theta_{\text {out }}=2 H+\frac{2 R}{(R+M)^{2}}, \quad \theta_{\text {in }}=2 H-\frac{2 R}{(R+M)^{2}} .
$$

It will be useful to define

$$
\alpha \equiv \sqrt{1+4 M H}
$$

and

$$
\beta \equiv \sqrt{1-4 M H}
$$

$\theta_{\text {out }}$ vanishes at

$$
R_{b h}=-\frac{1}{2 H}(1+2 M H-\alpha)
$$

and

$$
R_{d S}=-\frac{1}{2 H}(1+2 M H+\alpha),
$$

which correspond to the black hole and de Sitter horizons. The ingoing expansion 
$\theta_{\text {in }}$ vanishes at

$$
R_{\text {in }}=\frac{1}{2 H}(1-2 M H-\beta),
$$

which corresponds to the inner horizon. The quantities $\alpha$ and $\beta$ will play an important role in what follows.

In Fig. (5) we have drawn the coordinate patch covered by $(r, \tau)$. The coordinate patch is divided into four regions, labeled I - IV, by various horizons. These horizons coincide with the boundaries of the regions of trapped surfaces, $R_{b h}, R_{d S}, R_{i n}$, given in (3.10) - (3.12). Spheres in region I are outer trapped, and the boundary between regions I and II corresponds to $R_{d S}$. Spheres in region III are also outer trapped and the boundary between regions II and III is given by $R_{b h}$. Spheres in region IV are both inner and outer trapped.

We now consider the two-black-hole solution where each mass is less than the extremal limit $M_{i}<M_{e x t}$. The main difference from the one-black-hole case, or the multiple black holes with zero cosmological constant [9], is that these solutions are dynamical. The apparent horizon will evolve in time. Of course, with two black holes an apparent horizon will no longer be precisely spherical, but it will be approximately spherical in limiting cases. Hence we consider

$$
\theta_{\text {out }}=0,
$$

evaluated on appropriately centered spheres. (Trapped surfaces have been found for initial data describing two uncharged black holes with $\Lambda>0$ in [10].)

Spheres of sufficiently small radius, centered at $\vec{r}_{i}$, are always trapped. At early time, for $\tau \ll 0$, there is a solution for $\theta_{\text {out }}=0$ at $\left|\vec{r}-\vec{r}_{i}\right|=R_{b h}\left[M_{i}\right] / H \tau$, since the equation is identical to that of RNdS with mass $M_{i}$. (Here $R_{b h}\left[M_{i}\right]$ is defined as in (3.10), with $M$ replaced by $M_{i}$.) So, around each throat there is a region of outer trapped surfaces, surrounded by an external region. Actually, around each mass there is also the second solution to $\theta_{\text {out }}=0$ at $\left|\vec{r}-\vec{r}_{i}\right|=R_{d S}\left[M_{i}\right] / H \tau$. These 
two solutions correspond to the black hole horizon and the past de Sitter horizon in the RNdS spacetime.

Spheres of constant $r$ (where $r$ is much larger than the coordinate distance between the two masses) resemble spheres in the RNdS solution with mass equal to the total mass $M=\sum M_{i}$. If $M<M_{e x t}$, then at late times, as $\tau$ approaches zero, one again has two (outer) apparent horizons at $r=R_{b h} / H \tau$ and $r=R_{d S} / H \tau$. This suggests that the black holes coalesce. If $M>M_{\text {ext }}$, then there are no apparent horizons at late time for large $r$. We will have more to say about this case in Sec. 7.

One can understand the behavior of the apparent horizon as follows. For the RNdS solution, the horizon is at fixed $R$, which corresponds to $r \propto 1 / H \tau$. So when $\tau$ is large and negative, the horizon is at small $r$, and when $\tau \approx 0$, it is at large $r$. In both of these limits, the two black hole solution resembles the one black hole solution (although with different masses).

\section{Extensions}

\subsection{Locating the Horizons}

As one might expect from the RNdS solution, the region of spacetime described by the metric (3.1) (with $\tau, \vec{r}$ taking all real values) is incomplete, even away from the curvature singularity. It is bounded by the analog of the de Sitter horizon at large $r$ and the inner black hole horizon and past white hole horizon at small $\left|\vec{r}-\vec{r}_{i}\right|$. In both these regions the metric becomes approximately spherically symmetric. We will establish the incompleteness by considering radial null geodesics in these asymptotic regions.

We first derive an equation for the affine parameter $s$ along a radial null geodesic from the variational principle

$$
\delta \int\left(-\frac{1}{U^{2}}\left(\frac{d \tau}{d s}\right)^{2}+U^{2}\left(\frac{d r}{d s}\right)^{2}\right) d s=0
$$


Vary $\tau$ and use the outgoing null condition,

$$
d \tau / d r=U^{2}
$$

to find

$$
\frac{d^{2} r}{d s^{2}}+2 H U\left(\frac{d r}{d s}\right)^{2}=0
$$

If a null geodesic is known as $\tau=\tau(r)$, the affine parameter is given by quadratures,

$$
s=\int e^{2 H \int U(\tau(r), r) d r} d r
$$

In the limit of large $r$, the function $U$ takes the simple form

$$
U \rightarrow H \tau+\frac{M}{r}
$$

where $M=\sum M_{i}$. In this case, the null condition (4.2) is integrable when rewritten in terms of the variable $R$ of (3.6):

$$
\frac{d R}{d r}=R+H(R+M)^{2}
$$

We need only the asymptotic form of the solution. If $M<M_{e x t}$, then the right hand side has two roots at $R_{b h}$ and $R_{d S}$. Starting with $R>R_{b h}$ one finds that the solution always approaches $R_{d S}$ as $r \rightarrow \infty$ (see Fig. 6). Thus

$$
U(\tau(r), r) \rightarrow \frac{R_{d S}+M}{r}
$$

Using this in (4.4), and noting from (3.11) that $R_{d S}+M=-(1+\alpha) / 2 H$ we find that

$$
s \sim s_{H}+c r^{-\alpha}
$$

where $s_{H}$ is the horizon value of $s$, and $c$ is a constant of integration. Thus $s$ 
remains finite as $r \rightarrow \infty$. Asymptotically for large $r$, it follows that

$$
r \sim\left(s-s_{H}\right)^{-1 / \alpha}, \quad \tau \sim\left(s-s_{H}\right)^{1 / \alpha}
$$

This not only shows that these quantities reach their horizon values at a finite $s$, but also that $1 / r$ and $\tau$ are not smooth functions of $s$ (unless $1 / \alpha$ happens to be integral).

For each mass $M_{i}$, we can find similar results for ingoing null geodesics near the inner horizon, in coordinates centered about $M_{i}$. In the limit of small $r$, ingoing null geodesics satisfy

$$
\frac{d \tau}{d r}=-U^{2}=-\left(H \tau+\frac{M_{i}}{r}+\sum_{j \neq i} \frac{M_{j}}{r_{j}}\right)^{2}
$$

The last term on the right is a constant which can be removed by shifting the origin of $\tau$. We then obtain an equation like (4.6) whose solution has the limiting form

$$
R \rightarrow R_{i n}\left[M_{i}\right], \quad U(\tau(r), r) \rightarrow \frac{R_{i n}\left[M_{i}\right]+M}{r}
$$

Eq. (4.4) now implies that as $r$ goes to zero,

$$
s \sim s_{I}+C r^{\beta_{i}}
$$

where $s_{I}$ denotes the value of the affine parameter at the inner horizon, $C$ is a constant of integration and $\beta_{i} \equiv \sqrt{1+4 M_{i}|H|}$. So the affine parameter is again finite at the inner horizon. It follows that

$$
r \sim\left(s-s_{I}\right)^{1 / \beta_{i}}, \quad \tau \sim\left(s-s_{I}\right)^{-1 / \beta_{i}}
$$




\subsection{Extending Beyond the Horizons}

We now consider extensions across the horizons. We begin by introducing

new variables. For the single black hole, the static $\tilde{R}$ coordinate is good on the horizons. The closest analog for the solutions (3.1) is $R=H r \tau$ introduced above, where $r=0$ is chosen to correspond to the location of one of the masses. We also set

$$
y=\ln r, \quad W=r U \text {. }
$$

In $(R, y)$ coordinates, the metric (3.1) takes the form

$$
d s^{2}=-\frac{(d R-R d y)^{2}}{H^{2} W^{2}}+W^{2}\left(d y^{2}+d \Omega^{2}\right) .
$$

We have seen that the metric approaches the solution for a single black hole both in the limit of large and small $r$. However, in determining the behavior of the geometry across the horizon, the rate at which the metric approaches the singleblack-hole solution is crucial. We first show that all curvature scalars remain finite as one approaches these horizons. The $(R, y)$ part of the metric (4.15) has constant determinant. Thus the inverse metric has a similar form, with only $W^{2}$ appearing in the denominator. A general curvature scalar will involve terms consisting of derivatives of the metric and its inverse, multiplied by powers of the metric and its inverse. All of these terms reduce to derivatives of $W$ and $R$ divided by powers of $W$. But all derivatives of $W$ remain bounded as $y \rightarrow \pm \infty$, and since $W$ is finite on the horizon, these terms cannot blow up. (By simply shifting the origin of $r$, one can apply this argument to the horizons near each of the masses.)

It is tempting to conclude from this that the metric is smooth across the horizon and can be analytically continued as in the single-black-hole case. However this is incorrect. We will see that, in general, the horizon has only finite differentiability. The curvature can even be singular at the inner black hole horizon, but 
the singularity is "null," and so all curvature scalars remain finite ${ }^{\star}$. To establish this result, we will introduce coordinates that are good in a neighborhood of the horizon. It will turn out that $R$ is a good coordinate but that $r=e^{y}$ is not. (We have already seen in (4.9) and (4.13) that $r$ is not a smooth function of the affine parameter along null geodesics.) For the single-mass solution, $W=R+M$, so the metric (4.15) depends only on $R$, and therefore is smooth. However, the effect of the other masses is to modify the single-black-hole metric by a power series in $r$, so the exact metric is no longer smooth across the horizon.

The metric (4.15) can be rewritten in the form

$$
d s^{2}=\frac{1}{H^{2} W^{2}}\left[d R+\left(H W^{2}-R\right) d y\right]\left[-d R+\left(H W^{2}+R\right) d y\right]+W^{2} d \Omega^{2} .
$$

Our procedure for studying the extensions of the spacetime is to introduce new coordinates $(u, v)$ in the neighborhood of each horizon, such that the first quantity in brackets is proportional to $d u$ and the second is proportional to $d v$ at the horizon.

We first consider the de Sitter horizon $(y \rightarrow \infty)$. If we choose the origin of coordinates to be the center of mass, the metric takes the form (3.1) with

$$
U=H \tau+\frac{M}{r}+\frac{f(\theta, \phi)}{r^{3}}+O\left(r^{-4}\right)
$$

where $M$ is the sum of the individual masses. In terms of $(R, y)$ coordinates the metric takes the form (4.16) with

$$
W=R+M+f(\theta, \phi) e^{-2 y}+\cdots
$$

We saw in Sec. 4.1 that $R$ approaches the value $R_{d S}$ at the horizon. Expanding

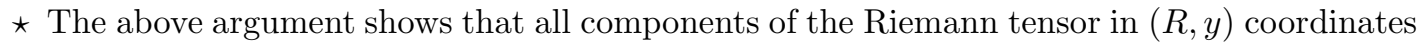
stay bounded, but this coordinate basis is not well behaved at the horizon.
} 
$H W^{2}$ about $R_{d S}$ yields

$$
H W^{2} \approx-R_{d S}-(1+\alpha)\left[R-R_{d S}+f e^{-2 y}\right]
$$

Define new coordinates

$$
\begin{gathered}
u=\left[1+\frac{\alpha}{2 R_{d S}}\left(R-R_{d S}\right)+\frac{\alpha(\alpha+1) f}{2 R_{d S}(\alpha+2)} e^{-2 y}\right] e^{-\alpha y} \\
v=-\left[R-R_{d S}+\frac{(\alpha+1) f}{\alpha-2} e^{-2 y}\right] e^{\alpha y} .
\end{gathered}
$$

The metric near the horizon becomes

$$
d s^{2}=\frac{2 R_{d S}}{\alpha H^{2} W^{2}} d u d v+W^{2} d \Omega^{2}
$$

So $u, v$ are good null coordinates near the horizon, which now corresponds to $u=0$. The curves $v=$ constant cross the horizon. The metric (4.21) depends on $W^{2}$ which involves factors of $\left(R-R_{d S}\right)$ and $e^{-2 y}$. In addition, there are corrections to the leading order behavior (4.21) that involve these same factors. To express these terms as functions of $u$ and $v$ we need to invert (4.20). Near the horizon we have $u=e^{-\alpha y}$, and $R-R_{d S}=-\left[v e^{-\alpha y}+(\alpha+1) f e^{-2 y} /(\alpha-2)\right]$. But since $\alpha=\sqrt{1-4 M|H|}<1$, as long as $v \neq 0$ the second term is negligible compared to the first as $y \rightarrow \infty$, and we have $R-R_{d S}=u v$. Thus corrections involving only powers of $R-R_{d S}$ will be smooth at the horizon. However, $e^{-y}=u^{1 / \alpha}$ is not smooth in general. Since the corrections start at order $e^{-2 y}=u^{2 / \alpha}$ and $\alpha<1$, we see that the metric is always at least $C^{2}$ in these coordinates. Since the metric is $C^{2}$, there is no curvature singularity. But one can show that in general, certain components of derivatives of the curvature diverge at the horizon, so there are no coordinates for which the metric is analytic. As a result, the extension across the horizon is not unique. One can match onto essentially any solution of the form (3.1) with the same total mass. (Similar behavior of finite differentiability across a horizon in an exact solution was found in [11].) 
The differentiability at the horizon can be increased in two ways. If the total mass $M$ is chosen so that $1 / \alpha$ is an integer $n$, then $e^{-y}$ is smooth and the metric is $C^{\infty}$. This occurs when

$$
4 M|H|=1-\frac{1}{n^{2}}
$$

For these values, the smooth continuation consists of matching the spacetime onto one with the same positions and magnitudes of all the masses so that all multipole moments agree, but with the opposite sign of $H$. We do not understand the physical significance of these special masses.

The second way to increase differentiability is to arrange the masses so that their first $n$ multipole moments vanish. Then the perturbation will begin at $e^{-n y}$. These solutions may provide a simple model of smoothness of null infinity in asymptotically flat spacetimes. It has been suggested [12] that the behavior of fields at null infinity may depend on their fall-off near spatial infinity. If the unconstrained part of the initial data falls off more quickly in spacelike directions, then perhaps the evolved fields will be more differentiable at null infinity. This is very similar to the behavior we find in the multi-black-hole solutions.

We now consider the inner horizon associated with one mass $M_{i}$ which we choose to be located at the origin. Near $r=0$ we have

$$
U=H \tau+\frac{M_{i}}{r}+c+g(\theta, \phi) r+\cdots
$$

where $c$ is a constant that can be removed by changing the origin of $\tau$. Thus $W=R+M_{i}+g r^{2}+\cdots$. We showed in Sec. 4.1 that $R$ approaches the constant $R_{\text {in }}\left[M_{i}\right]$ at the inner horizon. Expanding $H W^{2}$ near $R=R_{\text {in }}\left[M_{i}\right]$ yields:

$$
H W^{2} \approx R_{i n}\left[M_{i}\right]+\left(1-\beta_{i}\right)\left(R-R_{i n}\left[M_{i}\right]+g e^{2 y}\right)
$$

where, as before, $\beta_{i}=\sqrt{1+4 M_{i}|H|}$. Define new coordinates by

$$
u=\left[R-R_{i n}\left[M_{i}\right]+\left(\frac{1-\beta_{i}}{2-\beta_{i}}\right) g e^{2 y}\right] e^{-\beta_{i} y}
$$




$$
v=\left[1-\frac{\beta_{i}}{2 R_{i n}\left[M_{i}\right]}\left(R-R_{i n}\left[M_{i}\right]\right)+\frac{\beta_{i}\left(1-\beta_{i}\right)}{2 R_{i n}\left[M_{i}\right]\left(\beta_{i}+2\right)} g e^{2 y}\right] e^{\beta_{i} y}
$$

Then the leading order behavior of the metric near the horizon takes the simple form

$$
d s^{2}=\left(\frac{2 R_{i n}\left[M_{i}\right]}{\beta_{i} H^{2}}\right) \frac{d u d v}{W^{2}}+W^{2} d \Omega^{2}
$$

(The leading factor in parentheses is just a constant.) So $u, v$ are again good null coordinates near the horizon, which now corresponds to $v=0$. Near the horizon we have $v=e^{\beta_{i} y}$, and $R-R_{\text {in }}=u e^{\beta_{i} y}+\left(\beta_{i}-1\right) g e^{2 y} /\left(2-\beta_{i}\right)$. But since $\beta_{i}=\sqrt{1+4 M_{i}|H|}$, every black hole with $M_{i}<M_{\text {ext }}$ has $\beta_{i}<2$. Thus as long as $u \neq 0$ the second term is negligible compared to the first near the horizon, and we have $R-R_{i n}\left[M_{i}\right]=u v$. Corrections involving only powers of $R-R_{i n}\left[M_{i}\right]$ will again be smooth at the horizon. However, $r=e^{y}=v^{1 / \beta_{i}}$, and $1<\beta_{i}<2$. Thus $r$ is not smooth at the inner horizon. Since the corrections start at order $r^{2}$, the metric is $C^{1}$ but not $C^{2}$ in these coordinates.

To see that there are no better coordinates for which the metric is smooth, we compute a component of the curvature. Let $l=\partial / \partial v$ and $\eta=\partial / \partial \theta$. Then $R_{v \theta v \theta}=R_{\mu \nu \rho \sigma} l^{\mu} \eta^{\nu} l^{\rho} \eta^{\sigma}$ contains several terms that are finite at the horizon. But it contains one term which is infinite there. This is $g_{\theta \theta, v v}$, which involves two derivatives of $v^{2 / \beta_{i}}$. This divergence is null since it only occurs at $v=0$. We will return to the physical interpretation of this singularity shortly.

Unlike the case of the de Sitter horizon, there are no special values of the mass for which the inner horizon becomes smooth. However one can still increase the differentiability by carefully arranging the other masses so that the first $n$ powers of $r$ cancel in the expansion of $U$ in (4.23). In particular, one could remove the curvature singularity this way. However, the inner horizon associated with the other black holes will still be only $C^{1}$.

It is interesting to compare this situation with the case of zero cosmological constant. There, it was shown [13] that the spacetime describing several $Q=$ 
$M$ black holes has an analytic extension across each inner horizon, which simply corresponds to letting $r$ become negative. In other words, when $\Lambda=0, r$ is smooth at the horizon and there is no analog of the singularity we find above. This is consistent with our results since $\Lambda=0$ implies $\beta_{i}=1$ for all $i$, so $r=v$ at the inner horizon.

The remaining horizon is the past white hole horizon $r=0, \tau=-\infty$. One can show that the situation here is similar to the de Sitter horizon. The metric is always $C^{2}$ and can be made $C^{\infty}$ if the individual mass $M_{i}$ takes one of the discrete values (4.22) . Notice that if more than one mass has one of the discrete values, the total mass exceeds the extremal limit (3.2).

\subsection{Physical Interpretation of the Lack of Smoothness}

We now consider the physical interpretation of the lack of smoothness we have found at the horizons. Consider the solution with two black holes and take the limit where one mass becomes much less than the other. In this limit, we can think of the small mass as a test particle moving in the background of the large black hole. The single black hole solution is, of course, smooth everywhere. But the perturbation in the metric and Maxwell field obtained in this limit involves a function of $r$ (or $1 / r$ ), which is not smooth at the horizon. This seems rather unphysical. We would expect a $q=m$ test particle to radiate as it falls into the black hole, and the field it produces should remain smooth at the de Sitter horizon. This can be made more precise in terms of initial data. To avoid singularities associated with point particles, we can model the test particle by a small ball of $q=m$ dust. Let us take our initial surface to be the $T=0$ surface in the RNdS metric. One can certainly find smooth initial data for the linearized Einstein-Maxwell field equations that satisfy the constraints with the ball of dust as a source. The evolution of these initial data must be smooth everywhere in the domain of dependence, which

includes both the de Sitter horizon and past white hole horizon. The fact that the multi-black-hole solutions are not smooth at these horizons can be interpreted as saying that they describe more than colliding black holes. In addition, they contain 
a distribution of electromagnetic and gravitational radiation which is not smooth everywhere in the spacetime. We expect that there are other (undoubtedly more complicated) solutions describing colliding black holes that do not suffer from this lack of smoothness.

The singularity at the inner horizon is qualitatively different. This is because it is on the boundary of the domain of dependence of the initial data surface described above. The behavior we find is reminiscent of the instability of the inner black hole horizon which has been extensively studied for $\Lambda=0$ [4]. (When $\Lambda>0$ it has been argued that the inner horizon might be stable [5].) A key difference, however, is that the previous analyses were based on a perturbation expansion, while the solution (3.1) treats the effect of the other masses exactly. One unusual feature of these solutions is that only half of the Cauchy horizon becomes singular. The other half lies inside the $(\vec{r}, \tau)$ coordinates and is $C^{\infty}$. Perhaps this is related to the extra radiation in the spacetime that is responsible for the lack of smoothness at the de Sitter horizon.

The idea that the finite differentiability at the de Sitter and past white hole horizons is a result of additional radiation is supported by the fact that one can construct exact initial data for multiple charged black holes with $Q<M$. These initial data are smooth at the white hole horizon. This construction relies on the $\Lambda=0$ initial value solutions of Brill and Lindquist [14] for time-symmetric, arbitrarily placed wormholes of general mass and charge, and on the method of Nakao et al [10] to turn such a solution into one with a cosmological constant. The 3-metric and electromagnetic potential of [14] are

$$
\begin{gathered}
d s^{2}=(\chi \psi)^{2}\left(d x^{2}+d y^{2}+d z^{2}\right) \quad A=\nu \ln (\chi / \psi) \\
\chi=1+\sum_{i} a_{i} / r_{i}, \quad \psi=c+\sum_{i} b_{i} / r_{i},
\end{gathered}
$$

where $\nu$ is a unit normal form to the initial surface, and $a, b$ and $c$ determine the wormholes' masses and charges (approximately, $M_{i} \approx a_{i} / c+b_{i}, Q_{i} \approx-a_{i} / c+b_{i}$ ). 
Eq (4.27) satisfies the $\Lambda=0$ Einstein constraints if the extrinsic curvature $K_{i j}$ vanishes; therefore it satisfies the $\Lambda \neq 0$ constraints if we put [10]

$$
K_{i j}= \pm H g_{i j}, \quad H=\sqrt{\Lambda / 3}
$$

Special cases of (4.27) are initial values of the known dynamical solutions, as follows: The general $Q \neq M$ RNdS geometry in cosmological coordinates [8],

$$
\begin{gathered}
d s^{2}=-\frac{1-\frac{M^{2}-Q^{2}}{4 e^{2 H t} r^{2}} d t^{2}+e^{2 H t} U^{2}\left(d r^{2}+r^{2} d \Omega^{2}\right)}{U^{2}}+\frac{\left(1-\frac{M^{2}-Q^{2}}{4 R^{2}}\right)(d R-R d y)^{2}}{H^{2} W^{2}}+W^{2}\left(d y^{2}+d \Omega^{2}\right) \\
U=1+\frac{M}{e^{H t} r}+\frac{M^{2}-Q^{2}}{4 e^{2 H t} r^{2}} \quad W=R+M+\frac{M^{2}-Q^{2}}{4 R^{2}}
\end{gathered}
$$

has the form (4.27) with $a_{1}=(M-Q) / 2, b_{1}=(M+Q) / 2, a_{i}=0=b_{i}$ for $i \neq 1$, $c=1$, on the surface $t=0$. The solutions (3.1) have the form (4.27) with $a_{i}=0$, $b_{i}=m_{i}$, and $c=H \tau$.

We also note that the geometry of the surface $\tau=0$ of the solution (3.1), as well as that of the surface $\tilde{R}=M \pm \sqrt{M^{2}-Q^{2}}$ in the general RNdS geometry (in static coordinates) can be generalized by initial values of the type (4.27) with $c=0$. 


\section{Event Horizons}

All of the extensions considered above have a $\Im^{+}$beyond the "de Sitter horizon," which corresponds to the limit $(r, \tau) \rightarrow(\infty, 0)$, but $R \rightarrow R_{d S}$ finite, in our original coordinates (3.1). In these original coordinates we can therefore identify null curves that can go to $\Im^{+}$as those that reach this horizon. By contrast, the black hole horizon as defined in the Introduction (see also Ref. 7) is contained entirely within the original chart: it is the boundary of the region of events that can be causally connected to the de Sitter horizon. Thus we identify events from which outgoing null geodesics must reach the $U=0$ singularity as lying inside the black hole horizon.

In this section we establish several properties of null geodesics that have implications for the black hole horizon. For simplicity we confine attention to the solution (3.1) for two centers with identical mass parameter $M / 2$ with $M<M_{\text {ext }}$, separated in the 3D Euclidean base space by distance $2 a$. The origin of the Euclidean coordinate system is at the midpoint between the two centers. The Euclidean line connecting the two will be called the axis, and the perpendicular plane at the origin is the midplane. We show that for each sufficiently late (but negative) time $\tau$ there is a sphere of radius $r(\tau)$ such that all outgoing null geodesics from the sphere will reach the $U=0$ singularity - hence no causal curve from inside can reach $r=\infty$; but that there are points outside the sphere from which causal curves reach $r=\infty$ in finite affine parameter. That is, the sphere lies within the event horizon. We also show that at sufficiently early times, all points in the midplane can be connected to $r=\infty$ by causal curves; in other words, at early times the horizon does not meet the midplane. Finally, we will show that at sufficiently early times the event horizon consists of a sphere centered on each mass. Taken together these results prove that the black holes coalesce. The event horizon at early times has two disconnected pieces, but at late times it has only one.

We first consider the region inside the horizon. Every outgoing null geodesic 
must satisfy

$$
U^{2} d r^{2} \leq \frac{d \tau^{2}}{U^{2}}
$$

A lower limit on the "potential" $U$ is obtained by pretending that the total mass $M$ is concentrated at the greatest possible distance, $r+a$, so that

$$
\frac{d \tau}{d r} \geq U^{2}>\left(H \tau+\frac{M}{r+a}\right)^{2} .
$$

In terms of new variables $R_{\star}=H \tau(r+a), y_{\star}=\ln (r+a)$ the inequality simplifies,

$$
\frac{d R_{\star}}{d y_{\star}}<R_{\star}+H\left(R_{\star}+M\right)^{2} \equiv H\left(R_{\star}-R_{d S}\right)\left(R_{\star}-R_{b h}\right) .
$$

The slope of the function on the RHS is positive at its smaller zero, $R_{b h}$ (Fig. 6). Suppose that initially $R_{\star}<R_{b h}$, i.e., $a<r<\left(R_{b h} / H \tau\right)-a$. This can be fulfilled if $\tau>-R_{b h} / 2 a|H|$. Then $R_{\star}$ decreases with $r$ and becomes negative at a finite $r$. But then $\tau$ is positive, and as it increases further with $r$, the singularity at $U=0$ is reached at a finite $r$. Thus, as $\tau$ approaches zero (from below), there exist spheres of constant $r$ enclosing both masses such that all outgoing null geodesics hit the singularity.

We now show that at early times, a similar result holds for small spheres centered on each mass. For simplicity, let us shift the origin of spherical coordinates to be at one of the masses, and assume $r<a$. Then the "potential" from the other mass is at least $M / 6 a$, so that instead of $\mathrm{Eq}(5.2)$ we have

$$
\frac{d \tau}{d r} \geq U^{2}>\left[H\left(\tau+\frac{M}{6 a H}\right)+\frac{M}{2 r}\right]^{2} .
$$

Introducing the new variable $R_{+}=H r\left(\tau+\frac{M}{6 a H}\right)$ leads to an equation analogous to $(5.3)$,

$$
d R_{+} / d y<R_{+}+H\left(R_{+}+\frac{1}{2} M\right)^{2}
$$

Analyzing (5.5) in the same way as (5.3) we then find that events satisfying $R_{+}<$ $R_{b h}[M / 2]$ are inside the horizon. This inequality is satisfied at any early (negative) $\tau$ by all sufficiently small $r<R_{b h}[M / 2] /(H \tau+M / 6 a)$. 
To explore the outside of the black hole horizon, we ask under which conditions some causal curves from a given event can reach $r=\infty$. We first confine attention to the midplane, and obtain an upper limit on the inverse speed of purely radial null curves:

$$
\frac{d \tau}{d r}=U^{2}=\left(H \tau+\frac{M}{\sqrt{r^{2}+a^{2}}}\right)^{2}<\left(H \tau+\frac{\sqrt{2} M}{r+a}\right)^{2} .
$$

In terms of the variable $R_{\star}, y_{\star}$ introduced in Eq (5.3) this becomes

$$
d R_{\star} / d y_{\star}>R_{\star}+H\left(R_{\star}+\sqrt{2} M\right)^{2} .
$$

Arguing as before (see Fig. 6) we find that if $R_{\star}>R_{b h}[\sqrt{2} M]$ is satisfied initially for some $r$, then $R_{\star}$ will stay positive for all larger $r$. But this means that $\tau$ will remain negative as $r$ increases, avoiding the singularity.

For example, if the null curve starts at any $r$, but with $\tau<R_{b h}[\sqrt{2} M] / H a$, it satisfies the initial inequality and hence will be able to escape to $r=\infty$. Thus at early times no point in the midplane is within the horizon (provided of course that a real $R_{b h}[\sqrt{2} M]$ exists; if $M$ is close to the extremal limit, a more accurate investigation of the null geodesics is needed).

Similarly, for any $\tau<0$ we can find a sufficiently large

$$
r>\left(R_{b h}[\sqrt{2} M] / H \tau\right)-a
$$

so that $R_{\star}>R_{b h}[\sqrt{2} M]$ and null geodesics can escape to infinity. In fact, for sufficiently large $r$ the argument leading to the increase of $R_{\star}$ is valid also for points not on the midplane. Thus outside the sphere of events that must causally lead to the singularity there are events from which causal escape to infinity is possible. At early times, therefore, the event horizon surrounds each mass separately, and expands approximately according to $r=R_{b h}[M / 2] / H \tau$. At $\tau \approx R_{b h} / 2 a H$ the event horizon enters the midplane, and thereby changes its topology to a surface surrounding both centers. 


\section{Charged Shells and Dust}

As stated in the Introduction, in our test of cosmic censorship, we will need to introduce a shell of charged dust to remove an unwanted singularity. The dynamics of these shells is also of interest in its own right. For example, one can ask whether it is even possible to form $Q=M \mathrm{RNdS}$ black holes from collapsing matter. For $\Lambda=0$, Boulware [15] studied the dynamics of charged shells. He found that a shell having charge density equal to its rest mass density and also equal to its total mass density does not collapse. Rather, as one might expect, it stays at constant area. For $\Lambda>0$, on the other hand, we find that a simple extension of Boulware's calculation does give collapse to form $Q=M \operatorname{RNdS}$ black holes.

\subsection{SHELlS}

Consider a spherical charged shell in an otherwise empty spacetime with $\Lambda>0$. Inside the shell Birkhoff's theorem guarantees that the metric is de Sitter. Outside the shell, we match the metric to RNdS. In order to determine the motion of the

shell, one integrates Einstein's equation across the shell to obtain jump conditions on the curvature. Working in static coordinates, one finds that a shell having charge $Q$ equal to its rest mass $M$ also equal to its total mass (i.e. no kinetic energy) follows the same path (2.5) as the radially moving $q=m$ test particle discussed in Sec. 2.3. In cosmological coordinates then, the shells stay at constant comoving radius.

The physical picture of shell collapse is then quite simple. The metric has the form (3.1), with $U=H \tau$ for $r<r_{s}$ and $U=H \tau+M / r$ for $r>r_{s}$. The comoving radius of the horizon however, changes with time. For $M<M_{\text {ext }}$, there is a horizon at $R=H r_{H} \tau=R_{b h}$. For large negative $\tau, r_{H}$ is inside the shell and hence does not correspond to a horizon. As $\tau$ increases towards zero, $r_{H}$ sweeps past the shell, which is now contained within the black hole. The same process can be used for a number of $Q=M$ shells to form the multi-black-hole solutions, if the shells are located at an equipotential of $U$ and have a mass and charge distribution 
appropriate to a constant $U$ in the interior. Then the metric is de Sitter inside each shell, and the multi-black-hole metric outside. All the shells stay at constant comoving radius, and eventually become black holes which later merge. Spacetime diagrams for a single collapsing shell are given in Figs. (7a,b) for undermassive and overmassive shells. The shells take a finite cosmological time to reach the singularities, but an infinite proper time.

The shells in Fig. (7) all start on the right side of the Penrose diagram and collapse to the singularity on the left. We can also introduce shells on the left which collapse to the singularity on the right. This follows from the $T \rightarrow-T$ symmetry of the RNdS solution in static coordinates. The motion of these shells is simple in the $\left(r^{\prime}, \tau^{\prime}\right)$ coordinates defined in section 2.3. The shells stay at constant $r^{\prime}$. For $r^{\prime}<r_{s}^{\prime}$ the metric will be de Sitter, and for $r^{\prime}>r_{s}^{\prime}$ the metric will be RNdS. These shells will be useful for us in Sec. 7 in constructing our potential counterexample to cosmic censorship.

\subsection{DUST}

It turns out to be easy to handle arbitrary configurations of $q=m$ dust as well. Consider a general metric and gauge field of the form

$$
d s^{2}=\frac{1}{U^{2}} d \tau^{2}+U^{2} d \vec{r} \cdot d \vec{r}, \quad A_{\tau}=\frac{1}{U}
$$

where $\partial U / \partial \tau=H$. For matter consider charged, comoving dust. The matter stress-energy and current density are given by

$$
T_{a b}^{d u s t}=\rho_{\text {dust }} u_{a} u_{b}, \quad J_{a}=\rho_{\text {charge }} u_{a},
$$

where $u^{a}=\frac{1}{U}\left(\frac{\partial}{\partial t}\right)^{a}$ is the four velocity of the dust. The Hamiltonian constraint is 
given by

$$
\begin{aligned}
\mathcal{H} & =-{ }^{(3)} R+\frac{1}{g}\left(\pi^{i j} \pi_{i j}-\frac{1}{2} \pi^{2}\right) \\
& =4 \frac{\nabla^{2} U}{U^{3}}-\frac{2}{U^{4}} \delta^{i j} \partial_{i} U \partial_{j} U-6 H^{2} \\
& =-16 \pi\left(\rho_{\text {dust }}+\rho_{\text {Maxwell }}+\rho_{\text {cosmo }}\right) .
\end{aligned}
$$

The third term on the second line is just the cosmological term. The second is equal to the energy density of the Maxwell field. Thus, the first term must be compensated by the energy density of the dust. For a solution then, one requires

$$
\nabla^{2} U=-4 \pi \rho_{\text {dust }} U^{3}
$$

The Maxwell constraint gives the same equation (6.4), if $\rho_{\text {charge }}=\rho_{\text {dust }}$. The evolution equations require that $T_{i j}^{\text {dust }}=0$ and that $\partial_{\tau}\left(U^{3} \rho_{\text {dust }}\right)=0$. The first requirement is satisfied for comoving dust. The second condition requires that the function $\tilde{\rho}(\vec{x})=U^{3} \rho_{\text {dust }}$ be independent of $\tau$. The constraint equation (6.4) is then

$$
\nabla^{2} U=-4 \pi \tilde{\rho}
$$

We see that we can freely specify the function $\tilde{\rho}$. This, together with $\partial U / \partial \tau=$ $H$, and the boundary condition $U \rightarrow H \tau$ as $r \rightarrow \infty$ determine a unique $U$. The physical dust density $\rho_{\text {dust }}$ is then derived from $\tilde{\rho}$ and $U$. Note that the volume element $\sqrt{h}=U^{3}$ for the spatial metric, so that the total mass of a dust cloud is constant and given by

$$
M=\int d^{3} x \sqrt{h} \rho_{d u s t}=\int d^{3} x \tilde{\rho}
$$

Other measures of the dust cloud, e.g. its volume, are of course time dependent and consistent with the picture of a collapsing cloud. 


\section{Testing Cosmic Censorship}

Having developed the necessary properties of the multi-black-hole solution we now turn to our test of cosmic censorship. Consider two black holes, each with mass less than the extremal limit, but whose sum is greater. Since the multi-blackhole solutions are not spherically symmetric, it is difficult to describe their global structure completely. It is therefore convenient to focus on a two dimensional slice

of the spacetime. Consider first a curve which comes up one throat and down the second on a constant $\tau$ surface. Evolving in time, we obtain a two dimensional subset of the spacetime which is nonsingular for $\tau<0$, but develops a timelike singularity between the two throats at a certain time $\tau_{0}>0$ (when $U=0$ ). This appears to be a violation of cosmic censorship in which a naked singularity evolves from regular initial conditions. However, since this subset of the spacetime does not include the asymptotic region, one does not know if the singularity is hidden behind an event horizon.

A better choice is to consider the curve which starts at infinity and goes down one of the throats on a constant $\tau$ surface. Evolving in time, we obtain a two dimensional slice of the spacetime whose Penrose diagram is shown in Fig. (8a). For small $r$, the solution looks like a single subcritical black hole, except that $\Im^{+}$ has been pushed off to the future of the region shown. For large $r$, it resembles the overmassive case with its singularity. This singularity is naked and exists for all time. It is independent of whether the two black holes have collided or not, and is just a reflection of the fact that the total mass always was greater than the extremal limit. Fortunately, this singularity can be removed by adding a shell of charged matter. This follows from the fact that the solution, at large $r$, reduces to the overmassive RNdS solution, and we saw in Sec. 6.1 that the singularity in this solution could be removed by a shell. The Penrose diagram for the solution with the shell added in shown in Fig. (8b).

In the absence of a cosmological constant, one usually requires that a counterexample to cosmic censorship have nonsingular data on a surface that is asymp- 
totically flat outside of a compact set. In the presence of a cosmological constant, the analog would be nonsingular data on a compact manifold. Our example does not have a compact surface, but it has what might be considered "the next best thing." Consider the surface $S$ shown in Fig. (8b). This surface is defined by $\tau$ equal to a negative constant outside the shell, and any spacelike surface inside the shell that continuously joins to it. The initial data on this surface is nonsingular everywhere. The surface is not compact, but has two infinite throats (only one of which is shown on the figure). However, each of these throats is surrounded by a trapped surface. So one would not expect that the asymptotic regions down the throats could influence the solution in the interior.

The initial data on $S$ uniquely determines the solution up to the Cauchy horizon. As we have discussed in Sec. 4, the solution past the horizon is not unique but all extensions have a curvature singularity shown at the right in Fig. (8b). It is clear from the diagram that all observers originating from $\Im^{-}$reach the Cauchy horizon, and if they extend beyond, they will see the singularity. We have also seen in Sec. 4 that the horizon is not smooth. Generically, for the class of solutions (3.1), the horizon is $C^{1}$ but not $C^{2}$. If we end the spacetime at the horizon then, of course, there is no violation of cosmic censorship. But one can find choices of parameters such that the Cauchy horizon associated with one of the masses is at least $C^{2}$ and there is no curvature singularity. Furthermore, even in the general solution, geodesics can be extended beyond the horizon. So it seems reasonable to conclude that cosmic censorship is violated in these examples.

It is clear that this violation is associated with the infinite throats in the initial data. One way to see this is that in cases when the horizon is $C^{2}$ or smoother, it is homogeneous. There is no point where the curvature is becoming large, associated with the beginning of a naked singularity. The singularity seems to "come in from infinity." The fact that the asymptotic regions are hidden behind trapped surfaces in the initial data, does not seem to be sufficient to prevent the violation of cosmic censorship. In a sense, all of space collapses down the throats carrying all observers with it. 
In light of this, it is natural to ask whether cosmic censorship would be violated if one first formed the black holes from regular initial conditions. We have shown that one can, in fact, form the black holes using shells of charged dust. If one also removes the singularity at infinity with another shell as we have discussed above, one has compact initial data. The problem now is that the two types of shells collide in the spacetime before any singularities have formed. We do not know the solution explicitly after this occurs. However, even if naked singularities were found later in the evolution, one would not know whether they were fundamental, or artifacts of the dust approximation. It is well known that naked singularities can form in spherically symmetric dust collapse. These shell crossing or shell focusing singularities can also occur in the absence of gravity and hence have nothing to do with cosmic censorship.

\section{Conclusions}

Motivated by a new test of cosmic censorship, we have studied the global structure of the multi-black-hole solutions (3.1). We have found that, if the total mass is less than the extremal limit (3.2), then they describe black holes which coalesce. It is remarkable that an analytic solution describing coalescing black holes can be expressed in such a simple form. Somewhat surprisingly, we have also found that these solutions contain radiation which is not smooth at the de Sitter (and past white hole) horizon. Perhaps the presence of this radiation is related to the simplicity of the solution.

The test of cosmic censorship was based on the fact that there is an upper limit to the mass of a black hole when the cosmological constant is positive. We have seen that colliding two black holes which are each less than the extremal mass, but whose sum is greater, does produce naked singularities.

However, we cannot yet claim that this is a serious violation of cosmic censorship for two reasons. The first concerns how generic the violation is. As we have seen, the most general of the exact solutions has the naked singularity protected 
by a Cauchy horizon with a weak singularity. However, the exact solutions only describe a subset of black hole collisions with a positive cosmological constant. The initial position and masses can be specified arbitrarily but not their initial velocities. And, of course, one cannot specify arbitrary gravitational and electromagnetic radiation. It is not clear whether the most general solution has a Cauchy horizon with a stronger singularity. If so, then cosmic censorship would be preserved.

The other reason concerns the fact that the example involves eternal black holes and not ones which formed from compact initial conditions. It is not yet clear how physically reasonable collapse would affect the formation of naked singularities.

\section{Acknowledgments}

It is a pleasure to thank P. Chrusciel, J. Isenberg, V. Moncrief, and B. Schmidt for discussions. This work was supported in part by NSF Grants PHY-8904035, PHY-9008502 and NSF-THY-8714-684-A01. JT and DK thank the Institute for Theoretical Physics for its hospitality and support.

\section{Figure Captions}

Fig. 1: Penrose conformal diagram for the $Q=M$ RNdS geometry with $M<$ $M_{\text {ext }}$. The maximally extended spacetime continues indefinitely in all directions. The region covered by the cosmological coordinates $(\vec{r}, \tau)$ lies inside the bold lines. Two horizons are labeled. The inner horizon is the extension of the line labeled $r=\infty$ to the region between the singularities (also see Fig. 5). The solid curves represent $\tau=$ constant surfaces and the dotted curve shows a typical $r=$ constant surface. The dashed line denotes $\tau=0$.

Fig. 2: Penrose diagram for the $Q=M$ RNdS geometry with $M=M_{\text {ext }}$. The notation is the same as for Fig. 1.

Fig. 3: Penrose diagram for the $Q=M \operatorname{RNdS}$ geometry with $M>M_{\text {ext }}$. The notation is the same as for Fig. 1. 
Fig. 4: Qualitative representation of the geometry on spacelike surfaces of constant $\tau$ : (a) $\tau<0$, (b) $\tau=0$, (c) $0<\tau \ll \sum M_{i} / H a$, where $a$ is the typical coordinate distance between the centers, (d) $M_{i} / H a \ll \tau$.

Fig. 5: Regions of trapped surfaces and the horizons that separate them for the RNdS geometry.

Fig. 6: A plot of the function $F(R)=R+H(R+M)^{2}$. In determining the motion of radial null geodesics, one is lead to inequalities of the form $d R / d y<F(R)$ or $d R / d y>F(R)$. In the former case, it is clear that if $R<R_{b h}$ initially, it will continue to decrease and become negative. These curves must hit the singularity. In the latter case, if $R_{b h}<R<R_{d S}$ initially, then it must remain positive. These curves reach the de Sitter horizon. If equality holds, then $R$ approaches $R_{d S}$.

Fig. 7: The motion of a $Q=M$ shell in a RNdS geometry, (a) undermassive, (b) overmassive case. The shell follows a curve $r=$ constant $=r_{0}$. In the exterior region, $r>r_{0}$ (unshaded part of the diagram) the geometry is RNdS. In the interior (shaded, including the origin $r=0$ ) spacetime is homogeneous de Sitter space. The shell eliminates the singularity that would otherwise be present in the right half of the digram (cf. Figs 1 and 3). Shells that replace the singularity on the left by a de Sitter interior follow a curve $r^{\prime}=$ constant. The corresponding Penrose diagrams would be reflections of (a) and (b) about a vertical axis.

Fig. 8: (a) Penrose diagram for a two dimensional subspace of the two black hole solution. Each hole is undermassive, but their sum is overmassive. Near $r=\infty$, the left part of the diagram is similar to Fig. 3 (single mass with $M>M_{\text {ext }}$ ), and near $r=0$ the right half resembles Fig. 1 (single mass with $M<M_{\text {ext }}$ ). (b) Similar to (a) but with a shell replacing the singularity on the left. 


\section{REFERENCES}

1. R. Penrose, Rel. del Nuovo Cimento 1, 252 (1969).

2. R.M. Wald, Ann. Phys. 82, 548 (1974).

3. D. Kastor and J. Traschen, Phys. Rev. D47, 4476 (1993).

4. S. Chandrasekhar and J. Hartle, Proc. Roy. Soc. London A384, 301 (1982);

E. Poisson and W. Israel, Phys. Rev. D41, 1796 (1990); A. Ori, Phys. Rev. Lett. 67, 789 (1991).

5. F. Mellor and I. Moss, Phys. Rev. D41, 403 (1990).

6. T. Damour, in Proceedings of the Fourth Marcel Grossman Meeting, ed. R. Ruffini (North-Holland, Amsterdam, 1986) and references therein.

7. T. Shiromizu, K. Nakao, H. Kodama and K. Maeda, Phys. Rev. D47, R3099, (1993)

8. D. Brill and S. Hayward, Global Structure of a Black Hole Cosmos and Its Extremes, preprint gr-qc/9304007 (1993).

9. S.D. Majundar, Phys. Rev. 72, 930 (1947), A. Papapetrou, Proc. Roy. Irish Acad. A51, 191 (1947).

10. K. Nakao, K. Yamamoto and K. Maeda, Phys. Rev. D47, 3203 (1993)

11. P.T. Cruściel and D.B. Singleton, Commun. Math. Phys. 147, 137 (1992)

12. B. Schmidt, private communication

13. J.B. Hartle and S.W. Hawking, Comm. Math. Phys. 26, 87 (1972).

14. D.R. Brill and R.W. Lindquist, Phys. Rev. 131, 471 (1963)

15. D. Boulware, Phys. Rev. D8, 2363 (1973). 